\title{
Comparisons of Thyroid Hormone, Intelligence, Attention, and Quality of Life in Children with Obstructive Sleep Apnea Hypopnea Syndrome before and after Endoscopic Adenoidectomy
}

\author{
Hui-Wei Feng, ${ }^{1}$ Tao Jiang, ${ }^{2}$ Hong-Ping Zhang, ${ }^{1}$ Zhe Wang, ${ }^{1}$ Hai-Ling Zhang, \\ Hui Zhang, ${ }^{1}$ Xue-Mei Chen, ${ }^{1}$ Xian-Liang Fan, ${ }^{1}$ Yu-Dong Tian, ${ }^{3}$ and Tao Jia ${ }^{1}$ \\ ${ }^{1}$ Department of Otorhinolaryngology, The Second Hospital of Shandong University, Jinan 250000, China \\ ${ }^{2}$ Department of Otorhinolaryngology, Dezhou Municipal Hospital, Dezhou 253000, China \\ ${ }^{3}$ Department of Orthopedic Surgery, The First Hospital of Jinan, Jinan 250000, China
}

Correspondence should be addressed to Xian-Liang Fan; fanxianliang610@126.com

Received 9 July 2014; Revised 25 September 2014; Accepted 25 September 2014

Academic Editor: Peter S. Roland

Copyright (C) 2015 Hui-Wei Feng et al. This is an open access article distributed under the Creative Commons Attribution License, which permits unrestricted use, distribution, and reproduction in any medium, provided the original work is properly cited.

Objective. The aim of this study was to compare the differences in thyroid hormone, intelligence, attention, and quality of life (QoL) of children with obstructive sleep apnea hypopnea syndrome (OSAHS) before and after endoscopic adenoidectomy. Method. A total of 35 OSAHS children ( 21 males and 14 females with a mean age of $6.81 \pm 1.08$ years) were included in this study for analyzing the levels of thyroid hormone, intelligence, attention, and QoL. There were 22 children underwent endoscopic adenoidectomy with bilateral tonsillectomy (BT), while the other 13 children who underwent endoscopic adenoidectomy without bilateral tonsillectomy without BT. Results. Our results revealed no significant difference in serum free triiodothyronine (FT3), free thyroxine (FT4), and thyroid stimulating hormone (TSH) levels in OSAHS children before and after endoscopic adenoidectomy (all $P>0.05$ ). However, there were significant differences in full-scale intelligence quotient (FIQ) $(92.45 \pm 5.88$ versus 106.23 $\pm 7.39, P<0.001$ ), verbal intelligence quotient (VIQ) $(94.17 \pm 15.01$ versus $103.91 \pm 9.74, P=0.006)$, and performance intelligence quotient (PIQ) ( $94.12 \pm 11.04$ versus $104.31 \pm 10.05, P=0.001)$, attention $(98.48 \pm 8.74$ versus $106.87 \pm 8.58, P<0.001)$, and total OSA-18 scores $(87.62 \pm 17.15$ versus $46.61 \pm 10.15, P<0.001)$ between before and after endoscopic adenoidectomy in OSAHS children. Conclusion. Our findings provided evidence that the intelligence, attention, and QoL of OSAHS children may be significantly improved after endoscopic adenoidectomy.

\section{Introduction}

Obstructive sleep apnea hypopnea syndrome (OSAHS) is a respiratory disease caused by total or partial collapse on the upper airway [1]. It is characterized by recurrent airflow obstruction with symptoms and features such as excessive daytime sleepiness or hypersomnia, snoring, morning dry mouth upon awakening, and chest retraction during sleep [2]. With transient nocturnal hypoxia and poor quality sleep, pediatric OSAHS can negatively impact daytime alertness, school performance, behavior, and cardiovascular status and even lead to cognitive delays, hypertension, and heart failure
[3]. OSAHS, with its peak incidence between 2 and 8 years of age, can affect $2 \sim 3 \%$ of children $[4,5]$. However, snoring, the main symptom of pediatric OSAHS, is much more frequent ranging from $8 \%$ to $27 \%$ [6]. In Asia, the prevalence of pediatric OSAHS has ranged from $0 \%$ to $4.8 \%$ depending on the diagnosis criteria [7]. The etiology of OSAHS is multifactorial which is associated with hypertrophic adenoids, hypertrophic rhinitis, low soft palate, deviations of oral structures, and even hypothyroidism [8]. Comorbidities of OSAHS could be hypertension, insulin resistance, congestive heart failure, stroke, and cerebrovascular ischemic events [9]. Treatment of OSAHS can be divided into three 
categories, that is, conservative management, alleviation of airway obstruction, and surgery [10]. Lifestyle modification is a kind of conservative management which contains weight reduction, drugs and sedatives withdrawal, enough sleep, and sleeping positions adjustment [10]. As for alleviation of airway obstruction, continuous positive airway pressure and mandibular repositioning splints are preferred [11, 12]. However, surgeries such as tonsillectomy, tracheostomy, jaw advancement surgery, and maxilla-mandibular osteotomy are among the most common treatments of OSAHS with tonsillectomy highly effective in children $[13,14]$.

Adenotonsillectomy or adenoidectomy, by resecting tonsillar lymphoid tissue to remove the obstruction of the upper airway, is frequently performed for pediatric OSAHS [15]. Great improvements of thyroid function, intelligence, and attention and life quality of OSAHS patients after surgery have been observed by some studies, which can be indicators for tonsillectomy evaluation [16]. As one of the four indicators, the proposed relationship between hypothyroidism and OSAHS could be the deposition of mucoproteins in the upper airway, disturbances of the regulatory control of pharyngeal dilator muscles, and respiratory center depression [17]. And study has showed the recovery of OSAHS symptoms after hypothyroid replacement therapy [18]. Pediatric OSAHS may affect neurocognitive and behavioral domains of children, especially in attentional capacity and intelligence $[19,20]$. The disruption in the normal sleep architecture, hypoxemia, and hypercapnia caused by OSAHS could have an effect on prefrontal cortex lesions which play an important role in recognition and identification and finally affect children intelligence and attention [21]. The impact of OSAHS is not only limited to the disease symptoms alone, but also linked to life quality especially in activity limitation, emotions, and interpersonal relationships $[22,23]$. Due to the close connection between the four indicators and pediatric OSAHS, the surgery could have a great influence over the four indicators which in return can be the prediction for the valuation of the surgery. Therefore, we performed the present study to compare the differences in thyroid hormone, intelligence, attention, and quality of life (QoL) of children with OSAHS before and after endoscopic adenoidectomy, which could provide practical and reliable evaluation method for clinic diagnosis and treatment of OSAHS.

\section{Materials and Methods}

2.1. Ethics Statement. The study design was reviewed and approved by the Ethics Committee of the Second Hospital of Shandong University. The parents or guardians of all children had to sign informed consent in written form to undergo diagnostic and therapeutic procedure at the time of hospitalization.

2.2. Patients and Treatment. From September 2010 to June 2011, a total of 35 OSAHS children (21 males and 14 females with a mean age of $6.81 \pm 1.08$ years) with duration of 6 months to 2 years, and typical symptoms of restless sleep, snoring, apnea, mouth breathing, hyperactivity, sweating, night terrors, night crying, enuresis, daytime sleepiness and so on, were recruited at the Department of Otolaryngology of the Second Hospital of Shandong University. According to the Diagnoses Standard Clinical Practice Guideline: Diagnosis and Management of Childhood Obstructive Sleep Apnea Syndrome (draft) advanced by subcommittee on Chinese Journal of Otorhinolaryngology Head and Neck and Chinese Otolaryngology Research Team in Urumqi in 2007, all recruited children underwent overnight polysomnography for more than 7 hours and were proved to conform to diagnostic criteria. Children with previous otorhinolaryngologic surgery, acute or chronic renal diseases, hematological diseases, congenital heart diseases, inborn error of metabolism, cerebral palsy, and known intellectual disability due to a syndrome or hypothyroidism were not eligible to be enrolled into the study. All children with OSAHS underwent surgery using tracheal intubation under intravenous anaesthesia. Of these children, 22 children underwent bilateral tonsillectomy and endoscopic adenoidectomy, and the other 13 children underwent endoscopic adenoidectomy without bilateral tonsillectomy. All OSAHS children were followed up more than 6 months after surgery. Another 35 healthy children (17 males and 18 females; mean age $6.47 \pm 1.51$ years) without snore symptom were enrolled randomly as the control group during their visit to our pediatric outpatient clinic for routine physical examination.

2.3. Evaluation of Thyroid Hormone Concentration. The serum of OSAHS children was collected preoperatively and postoperatively. Serum free triiodothyronine (FT3), free thyroxine (FT4), and thyroid stimulating hormone (TSH) levels were measured in the morning for 4 weeks using the electrochemiluminescence immunoassay (ECLIA) method (Roche Diagnostics GmbH, Basel, Switzerland) twice, before the treatment and after hormones stabilization. The reference ranges for FT3, FT4, and TSH are 3.0 9.0 pmol/L, 10.3 $25.8 \mathrm{pmol} / \mathrm{L}$, and $0.25 \sim 5.0 \mu \mathrm{IU} / \mathrm{mL}$, respectively.

2.4. Quality of Life (QoL) Assessment. The disease specific QoL for children with obstructive sleep apnea 18-item survey (OSA-18) was used to measure QoL in children with OSAS and improvement in QoL after the treatment [24]. We used OSA-18 to assess QoL before and 6 months after treatment in five domains, including sleep-disordered breathing, physical symptoms, emotions, daytime behaviors, and impact of guardians. The OSA-18, consisting of 18 relevant items, is scored based on seven levels regarding the frequency of symptoms: (1) never: 1; (2) hardly: 2; (3) seldom: 3; (4) sometimes: 4; (5) frequently: 5; (6) mostly: 6; (7) absolutely: 7. Total OSA-18 scores range from 18 (lowest) to 126 (highest). According to the OSA-18 scores, the included subjects were classified into three levels: mild OSAS $(<60)$, moderate OSAS $(60-80)$, and sever OSAS (>80), respectively. In addition, the improvement in QoL after the treatment was assessed with change in OSA-18 scores, which was measured by the differences of preoperative average OSA-18 scores (OSA-18 total score/18) with postoperative average OSA-18 scores, ranging from -7.0 to +7.0. A negative value indicates deterioration, while a positive value indicates improvement, which was further divided into 4 degrees: $(1)<0.5$, slight improvement; 
TABLE 1: Baseline characteristics of children with obstructive sleep apnea hypopnea syndrome (OSAHS) and healthy children.

\begin{tabular}{|c|c|c|c|c|}
\hline Characteristic & OSAHS group $(n=35)$ & Control group $(n=35)$ & $\chi^{2} / t$ & $P$ value \\
\hline Age (years) & $6.81 \pm 1.08$ & $6.47 \pm 1.51$ & 0.214 & 0.832 \\
\hline Gender (male/female) & $21 / 14$ & $17 / 18$ & 0.921 & 0.337 \\
\hline Body mass index (BMI) & $22.58 \pm 3.22$ & $22.49 \pm 2.89$ & 0.897 & 0.372 \\
\hline Height $(\mathrm{cm})$ & $1.06 \pm 0.20$ & $1.07 \pm 0.27$ & 1.470 & 0.293 \\
\hline Weight $(\mathrm{kg})$ & $25.41 \pm 4.36$ & $25.70 \pm 3.55$ & 0.325 & 0.747 \\
\hline Bone age & $-0.51 \pm 0.21$ & $-0.02 \pm 0.01$ & 1.789 & 0.038 \\
\hline Osteocalcin $(\mu \mathrm{g} / \mathrm{L})$ & $7.41 \pm 1.31$ & $10.22 \pm 2.50$ & 2.142 & 0.037 \\
\hline
\end{tabular}

TABLE 2: Thyroid hormone, intelligence, attention, and quality of life of OSAHS children before and after endoscopic adenoidectomy and healthy children.

\begin{tabular}{|c|c|c|c|c|c|c|c|c|}
\hline Group & $N$ & $\mathrm{FT}_{3}(\mu \mathrm{mol} / \mathrm{L})$ & $\mathrm{FT}_{4}(\mu \mathrm{mol} / \mathrm{L})$ & TSH (uIU/mL) & FIQ & VIQ & PIQ & OSA-18 \\
\hline \multicolumn{9}{|l|}{ OSAHS group } \\
\hline Before surgery & 35 & $6.04 \pm 1.88$ & $14.17 \pm 5.01$ & $4.12 \pm 1.04$ & $92.45 \pm 5.88$ & $94.17 \pm 15.01$ & $94.12 \pm 11.04$ & $87.62 \pm 17.15$ \\
\hline After surgery & 26 & $6.23 \pm 2.39$ & $13.91 \pm 4.74$ & $4.31 \pm 1.05$ & $106.23 \pm 7.39^{*}$ & $103.91 \pm 9.74^{*}$ & $104.31 \pm 10.05^{*}$ & $46.61 \pm 10.15^{*}$ \\
\hline With BT & 22 & $6.46 \pm 1.73$ & $12.81 \pm 3.26$ & $4.07 \pm 0.88$ & $112.05 \pm 6.87^{*}$ & $109.22 \pm 8.18^{*}$ & $109.54 \pm 9.97^{*}$ & $39.89 \pm 11.23^{*}$ \\
\hline Without BT & 13 & $6.12 \pm 3.06$ & $14.09 \pm 4.91$ & $4.67 \pm 1.32$ & $103.14 \pm 8.13^{*}$ & $98.77 \pm 10.27$ & $101.28 \pm 11.83^{*}$ & $51.24 \pm 9.64^{*}$ \\
\hline Control group & 35 & $6.21 \pm 2.45$ & $14.21 \pm 4.63$ & $4.33 \pm 1.29$ & $110.59 \pm 8.32^{*}$ & $106.91 \pm 11.25^{*}$ & $109.37 \pm 7.91^{*}$ & $37.81 \pm 8.67^{*}$ \\
\hline
\end{tabular}

$\mathrm{FT}_{3}$ : free triiodothyronine; $\mathrm{FT}_{4}$ : free thyroxine; TSH: thyroid stimulating hormone; FIQ: full-scale intelligence quotient; VIQ: verbal intelligence quotient; PIQ: performance intelligence quotient; ${ }^{*} P<0.05$ compared with the levels before surgery.

(2) $0.5-0.99$, mild improvement; (3) $1.00-1.49$, moderate improvement; and (4) $\geq 1.5$, obvious improvement.

\subsection{Cognition and Attention Assessment. General intellectual} functioning was assessed by a gold standard instrument, the Chinese Wechsler Intelligence Scale for Children (C-WISC), which is applicable for children ranging in age from 6 to 16 years. All tests were performed in a separated and quiet room by a trained psychiatrist. The C-WISC is composed of 11 distinct subtests divided into two scales, a verbal scale (VS) and a performance scale (PS). The VS includes information (I), comprehension (C), picture generalization $(\mathrm{PG})$, arithmetic (A), and picture and vocabulary (PV); and PS consists of picture arrangement (PA), picture completion (PC), block design (BD), animal egg (AE), maze analysis (MA), geometric design (GD), and vision analysis (VA). The verbal intelligence quotient (VIQ) was measured by VS that are language based items, performance intelligence quotient (PIQ) was measured by PS that are visual motor items and are less dependent on language, and full intelligence quotient (FIQ) was measured by full scale (FS). Before and 6 months after treatment, eleven of the subtests in each scale were assessed to produce scale-specific intelligence quotients (IQs) such as VIQ, PIQ, and FIQ (range from 85 to 115 in normal subjects), as well as concentration factor (factor $\mathrm{C}$ ).

2.6. Statistical Analysis. Data are presented as mean \pm standard deviation. The $t$-test was applied in the comparison of thyroid hormone, intelligence, attention, and QoL between before and after endoscopic adenoidectomy. Further comparison among the three groups was performed using the analysis of variance (ANOVA). All statistical analyses were conducted using the SPSS 17.0 software (SPSS Inc., Chicago,
Illinois, USA). A $P$ value of $<0.05$ was considered statistically significant.

\section{Results}

3.1. Baseline Characteristics. There was no significant difference in age, gender, body mass index (BMI), height, and weight between OSAHS and healthy children (all $P>0.05$ ). However, significant differences were observed in osteocalcin (7.41 \pm 1.31 versus $10.22 \pm 2.50, P=0.037)$ and the bone age $(-0.51 \pm 0.21$ versus $-0.02 \pm 0.01, P=0.038)$. The baseline characteristics of the individual subject were presented in Table 1.

3.2. Changes of Thyroid Hormone, Intelligence, Attention, and Quality of Life before and after Endoscopic Adenoidectomy. As shown in Table 2, there were no significant differences in serum FT3, FT4, and TSH levels in OSAHS children before and after endoscopic adenoidectomy (all $P>0.05$ ). However, there were significant differences in FIQ $(92.45 \pm 5.88$ versus $106.23 \pm 7.39, P<0.001)$, VIQ $(94.17 \pm 15.01$ versus $103.91 \pm$ 9.74, $P=0.006)$, and PIQ (94.12 \pm 11.04 versus $104.31 \pm 10.05$, $P=0.001)$, attention $(98.48 \pm 8.74$ versus $106.87 \pm 8.58$, $P<0.001)$, and total OSA-18 scores $(87.62 \pm 17.15$ versus $46.61 \pm 10.15, P<0.001)$ between before and after endoscopic adenoidectomy in OSAHS children. Furthermore, we also observed differences in thyroid hormone, intelligence, attention, and QoL between OSAHS children before surgery and healthy children (all $P<0.05$ ).

3.3. Comparisons of Thyroid Hormone, Intelligence, Attention, and Quality of Life between OSAHS Children with and without Bilateral Tonsillectomy. The present study demonstrated that OSAHS children with BT had higher FIQ, VIQ, and PIQ 
and lower OSA-18 scores than those of OSAHS children without BT (all $P<0.05$ ). Nevertheless, we also found no significant differences in serum FT3, FT4, and TSH levels between OSAHS children with BT and those without BT (all $P>0.05)$.

\section{Discussion}

In this present study, we aimed to explore the differences in thyroid hormone, intelligence, attention, and QoL of children with obstructive sleep apnea hypopnea syndrome (OSAHS) before and after endoscopic adenoidectomy. The findings in our study have revealed that the intelligence, attention, and QoL in children with OSAHS after surgery were significantly improved compared with those before surgery, suggesting that the intelligence, attention, and QoL of OSAHS children may be significantly improved after endoscopic adenoidectomy. It has been well known that OSAHS may result in various kinds of pathophysiological consequence including hypertension, functional impairments of heart, polycythemia, and lesions in many other systems and when present it may contribute to worse outcomes in human bodies [25]. Owing to these symptoms and functional impairments, patients with OSAHS, especially children, usually suffer from cognitive impairment and have a poor QoL from physical and emotional health to social functioning [26]. Children with OSAHS may experience several neurobehavioral problems, such as attention deficit, learning problems, memory deficits, hyperactive, behavioral disorders, and hypersomnolence, and it has also been linked to lower childhood IQ scores [27]. Many researchers have claimed that sleep-related breathing disorders including OSAHS, high microarousal index, intermittent hypoxia, and alterations in the sleep architecture may result in insufficient growth hormone, impairment of cognitive function, abnormalities of behavior and psychology, and so on [27, 28]. Among children, these complications are frequently noted clinically as decreased memory and learning abilities, aprosexia, hypomnesia, impairment of memory function, mania, and depression [29]. Moreover, OSAHS is often associated with oxygen deficit and metabolic disturbance which may result in cognitive impairment, abnormalities of behavior and psychology [26]. A previous study performed by Yang et al. has revealed that intermittent hypoxia resulting from OSAHS may cause structural neuron damage and dysfunction in the central nervous system, especially in the hippocampus, which can be regarded as potential mechanisms of neurocognitive and behavioral deficits [30].

The main finding of this study demonstrates that the scores of FIQ, VIQ, and PIQ in OSAHS children were obviously decreased after the operation when compared with the scores in preoperative children with OSAHS, suggesting that operation can improve the response control, attention, and hyperactivity behaviors of children with OSAHS. In addition, the continuous performance test revealed that factor $\mathrm{C}$ was significantly increased in the patients with OSAHS after operation in comparison with those before operation, implying that the early surgical treatment for OSAHS can improve the symptom of cognitive impairment and enhance attention of children. As an attention/vigilance factor, factor $\mathrm{C}$ can reflect some characteristics of attention impairment and closely relates to the school performance in children $[19,31]$. Thus, the increased factor $\mathrm{C}$ may indicate that surgical treatment of OSAHS children can promote the recoveries of attention impairment and further improve the school performance of children. As stated above, children with OSAHS often suffer from obviously decreasing cognitive function including lower learning abilities, decreased memory performance, and aprosexia, which may largely influence the QoL; thus children with OSAHS frequently have poor QoL. In this regard, we suspected that the improvement and recovery of intelligence and attention in OSAHS children may lead to the improvement of QoL. In this study, we adopted OSA-18 to assess the QoL in children with OSAHS from five parts including sleep-disordered breathing, physical symptoms, emotions, daytime behaviors, and impact of guardians [24]. Our results have revealed that the total OSA-18 scores after operation were significantly decreased compared with the preoperative total OSA-18 scores in children with OSAHS, suggesting that the QoL in children may be affected by OSAHS, and the surgical treatment can improve the QoL in children.

Thyroid hormone is primarily responsible for regulation of metabolism, which can increase the basal metabolic rate, affect protein synthesis, and regulate long bone growth (cooperate with growth hormone) and neural maturation $[32,33]$. Also, it has been reported that the thyroid hormone plays crucial roles in the cells proliferation and differentiation in human body, and various physiological and pathological stimuli may influence thyroid hormone synthesis [34, 35]. However, a study performed by Lanfranco $\mathrm{F}$ has demonstrated that obese patients with OSAHS were not obviously related to impaired thyroid activity in either basal conditions or an altered TSH response to TRH challenge test [36]. In consistent with previous study and document, our study also found that there was no significant difference between the serum levels of T4, FT3, and TSH in OSAHS children in pretreatment and posttreatment periods. The possible reason for this outcome may be that the severity of intermittent hypoxia caused by OSAHS cannot induce the decreased secretion of thyroid hormone. One limitation of the studies that assessed the changes of serum levels of T4, FT3, and TSH in OSAHS children is that small sample size in our study and all OSAHS children were not well grouped. We need to conduct further studies to explore the relationship between the severe OSAHS and thyroid function in children.

In summary, our findings provided evidence that the intelligence, attention, and QoL of OSAHS children may be significantly improved after endoscopic adenoidectomy. However, due to many limitations, our conclusion should be confirmed in a larger prospective trial.

\section{Conflict of Interests}

The authors have declared that no conflict of interests exists.

\section{Acknowledgments}

This work is funded by the Project of Science and Technology Planning Project of Shandong Province (no. 
2007GG30008004). The authors would like to acknowledge the reviewers for their helpful comments on this paper.

\section{References}

[1] R. Hornero, D. Alvarez, D. Abasolo, F. del Campo, and C. Zamarron, "Utility of approximate entropy from overnight pulse oximetry data in the diagnosis of the obstructive sleep apnea syndrome," IEEE Transactions on Biomedical Engineering, vol. 54, no. 1, pp. 107-113, 2007.

[2] N. B. Jalandhara, A. Patel, R. R. Arora, and P. Jalandhara, "Obstructive sleep apnea: a cardiopulmonary perspective and medical therapeutics," American Journal of Therapeutics, vol. 16, no. 3, pp. 257-263, 2009.

[3] S. R. Parikh, B. Sadoughi, S. Sin, S. Willen, K. Nandalike, and R. Arens, "Deep cervical lymph node hypertrophy: a new paradigm in the understanding of pediatric obstructive sleep apnea," The Laryngoscope, vol. 123, no. 8, pp. 2043-2049, 2013.

[4] I. E. Tapia and C. L. Marcus, "Newer treatment modalities for pediatric obstructive sleep apnea," Paediatric Respiratory Reviews, vol. 14, no. 3, pp. 199-203, 2013.

[5] B. S. Chhangani, T. Melgar, and D. Patel, "Pediatric obstructive sleep apnea," Indian Journal of Pediatrics, vol. 77, no. 1, pp. 81-85, 2010.

[6] D. Gozal, "Morbidity of obstructive sleep apnea in children: facts and theory," Sleep and Breathing, vol. 5, no. 1, pp. 35-42, 2001.

[7] T. Kitamura, S. Miyazaki, H. Kadotani et al., "Prevalence of obstructive sleep apnea syndrome in Japanese elementary school children aged 6-8 years," Sleep and Breathing, vol. 18, no. 2, pp. 359-366, 2014.

[8] Y. Zhao, L. Tao, P. Nie et al., "Association between 5-HT2A receptor polymorphisms and risk of obstructive sleep apnea and hypopnea syndrome: a systematic review and meta-analysis," Gene, vol. 530, no. 2, pp. 287-294, 2013.

[9] H. Hattori, C. Hattori, A. Yonekura, and T. Nishimura, "Two cases of sleep apnea syndrome caused by primary hypothyroidism," Acta Oto-Laryngologica, no. 550, pp. 59-64, 2003.

[10] G. Mbata and J. Chukwuka, "Obstructive sleep apnea hypopnea syndrome," Annals of Medical and Health Sciences Research, vol. 2, no. 1, pp. 74-77, 2012.

[11] C. McDaid, S. Griffin, H. Weatherly et al., "Continuous positive airway pressure devices for the treatment of obstructive sleep apnoea-hypopnoea syndrome: a systematic review and economic analysis," Health Technology Assessment, vol. 13, no. 4, pp. 1-119, 143-274, 2009.

[12] M. P. Villa, S. Miano, and A. Rizzoli, "Mandibular advancement devices are an alternative and valid treatment for pediatric obstructive sleep apnea syndrome," Sleep and Breathing, vol. 16, no. 4, pp. 971-976, 2012.

[13] M. P. Villa, R. Castaldo, S. Miano et al., "Adenotonsillectomy and orthodontic therapy in pediatric obstructive sleep apnea," Sleep and Breathing, vol. 18, no. 3, pp. 533-539, 2014.

[14] T. F. Hoban, "Obstructive sleep apnea in children," Current Treatment Options in Neurology, vol. 7, no. 5, pp. 353-361, 2005.

[15] Q. Zhang, D. Li, and H. Wang, "Long term outcome of tonsillar regrowth after partial tonsillectomy in children with obstructive sleep apnea," Auris Nasus Larynx, vol. 41, no. 3, pp. 299-302, 2014.

[16] P. S. Roland, R. M. Rosenfeld, L. J. Brooks et al., "Clinical practice guideline: polysomnography for sleep-disordered breathing prior to tonsillectomy in children," Otolaryngology-Head and Neck Surgery, vol. 145, no. 1, pp. S1-S15, 2011.

[17] S. A. Bahammam, M. M. Sharif, A. A. Jammah, and A. S. Bahammam, "Prevalence of thyroid disease in patients with obstructive sleep apnea," Respiratory Medicine, vol. 105, no. 11, pp. 1755-1760, 2011.

[18] J. M. Neal and R. J. O. Yuhico, ““Myxedema madness” associated with newly diagnosed hypothyroidism and obstructive sleep apnea," Journal of Clinical Sleep Medicine, vol. 8, no. 6, pp. 717718, 2012.

[19] B. Tulek, N. B. Atalay, F. Kanat, and M. Suerdem, "Attentional control is partially impaired in obstructive sleep apnea syndrome," Journal of Sleep Research, vol. 22, no. 4, pp. 422-429, 2013.

[20] J. Feng, Q. Wu, D. Zhang, and B.-Y. Chen, "Hippocampal impairments are associated with intermittent hypoxia of obstructive sleep apnea," Chinese Medical Journal, vol. 125, no. 4, pp. 696-701, 2012.

[21] X.-H. Cai, X.-C. Li, Q.-Q. Hu et al., "Multiple system morbidities associated with children with snore symptom," Pediatric Pulmonology, vol. 48, no. 4, pp. 381-389, 2013.

[22] I. W. Seetho and J. P. H. Wilding, "Screening for obstructive sleep apnoea in obesity and diabetes-potential for future approaches," European Journal of Clinical Investigation, vol. 43, no. 6, pp. 640-655, 2013.

[23] J. M. Wood, M. Cho, and A. S. Carney, "Role of subtotal tonsillectomy ("tonsillotomy") in children with sleep disordered breathing," Journal of Laryngology and Otology, vol. 128, no. 1, pp. S3-S7, 2014.

[24] R. A. Franco Jr., R. M. Rosenfeld, and M. Rao, "Quality of life for children with obstructive sleep apnea," Otolaryngology: Head and Neck Surgery, vol. 123, no. 1 I, pp. 9-16, 2000.

[25] F. B. Nerbass, R. P. Pedrosa, N. J. Danzi-Soares, L. F. Drager, E. Arteaga-Fernández, and G. Lorenzi-Filho, "Obstructive sleep apnea and hypertrophic cardiomyopathy: a common and potential harmful combination," Sleep Medicine Reviews, vol. 17, no. 3, pp. 201-206, 2013.

[26] M. Grigg-Damberger and F. Ralls, "Cognitive dysfunction and obstructive sleep apnea: from cradle to tomb," Current Opinion in Pulmonary Medicine, vol. 18, no. 6, pp. 580-587, 2012.

[27] S. Miano, M. C. Paolino, A. Urbano et al., "Neurocognitive assessment and sleep analysis in children with sleep-disordered breathing," Clinical Neurophysiology, vol. 122, no. 2, pp. 311-319, 2011.

[28] Y. E. Landau, O. Bar-Yishay, S. Greenberg-Dotan, A. D. Goldbart, A. Tarasiuk, and A. Tal, "Impaired behavioral and neurocognitive function in preschool children with obstructive sleep apnea," Pediatric Pulmonology, vol. 47, no. 2, pp. 180-188, 2012.

[29] R. B. Mitchell and J. Kelly, "Behavior, neurocognition and quality-of-life in children with sleep-disordered breathing," International Journal of Pediatric Otorhinolaryngology, vol. 70, no. 3, pp. 395-406, 2006.

[30] Q. Yang, Y. Wang, J. Feng, J. Cao, and B. Chen, "I ntermittent hypoxia from obstructive sleep apnea may cause neuronal impairment and dysfunction in central nervous system: the potential roles played by microglia," Neuropsychiatric Disease and Treatment, vol. 9, pp. 1077-1086, 2013.

[31] R. L. Ownby and C. G. Matthews, "On the meaning of the WISC-R third factor: relations to selected neuropsychological measures," Journal of Consulting and Clinical Psychology, vol. 53, no. 4, pp. 531-534, 1985. 
[32] B. Kim, "Thyroid hormone as a determinant of energy expenditure and the basal metabolic rate," Thyroid, vol. 18, no. 2, pp. 141-144, 2008.

[33] G. R. Williams, "Actions of thyroid hormones in bone," Endokrynologia Polska, vol. 60, no. 5, pp. 380-388, 2009.

[34] P. Iglesias and J. J. Díez, "Thyroid dysfunction and kidney disease," European Journal of Endocrinology, vol. 160, no. 4, pp. 503-515, 2009.

[35] T. K. Roepke, E. C. King, A. Reyna-Neyra et al., "Kcne2 deletion uncovers its crucial role in thyroid hormone biosynthesis," Nature Medicine, vol. 15, no. 10, pp. 1186-1194, 2009.

[36] F. Lanfranco, "Sleep apnea syndrome and hypothyroidism," Endocrine, vol. 44, no. 3, pp. 551-552, 2013. 


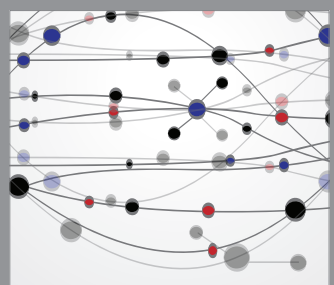

The Scientific World Journal
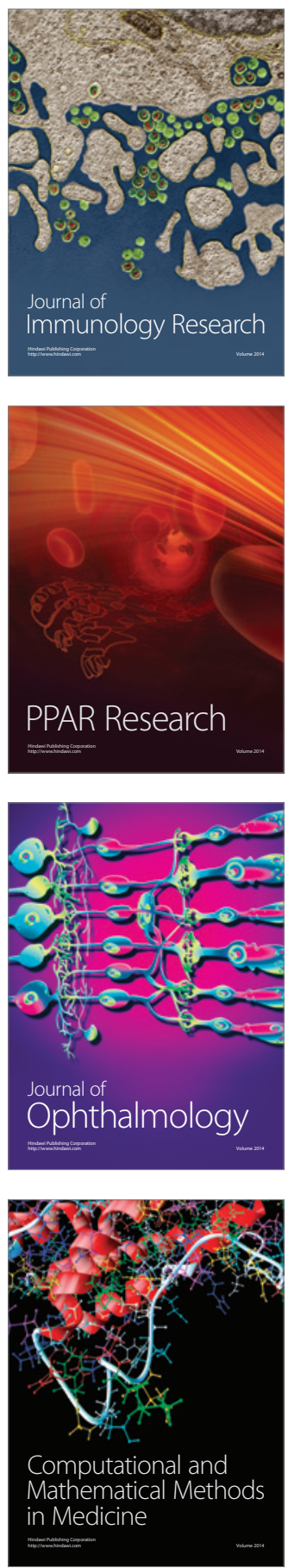

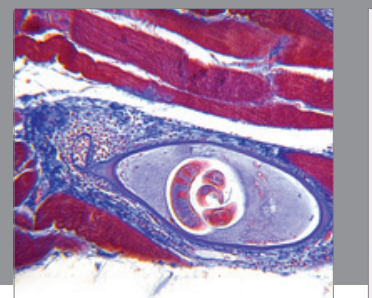

Gastroenterology

Research and Practice
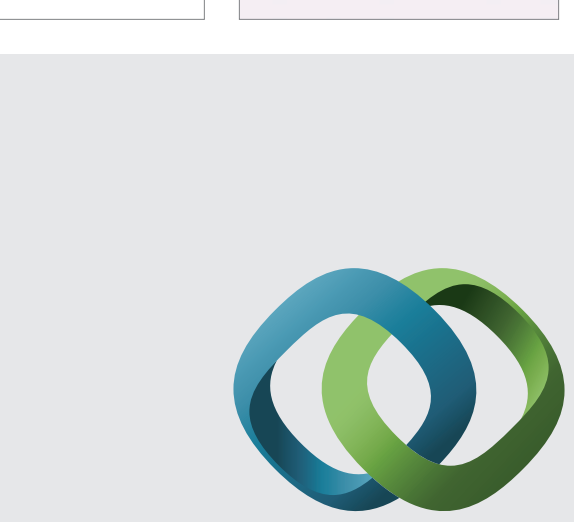

\section{Hindawi}

Submit your manuscripts at

http://www.hindawi.com
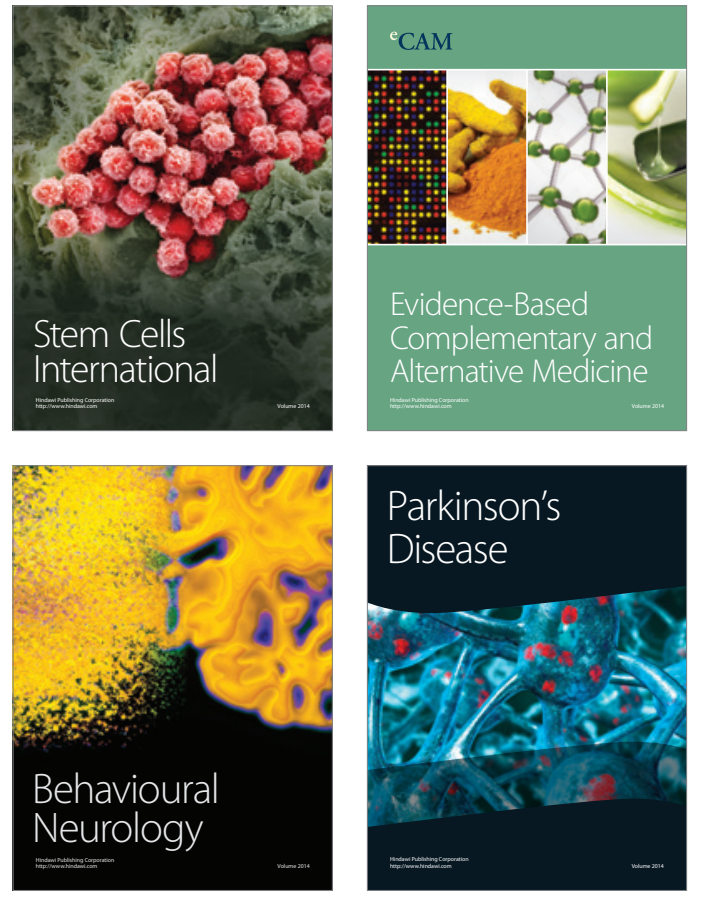
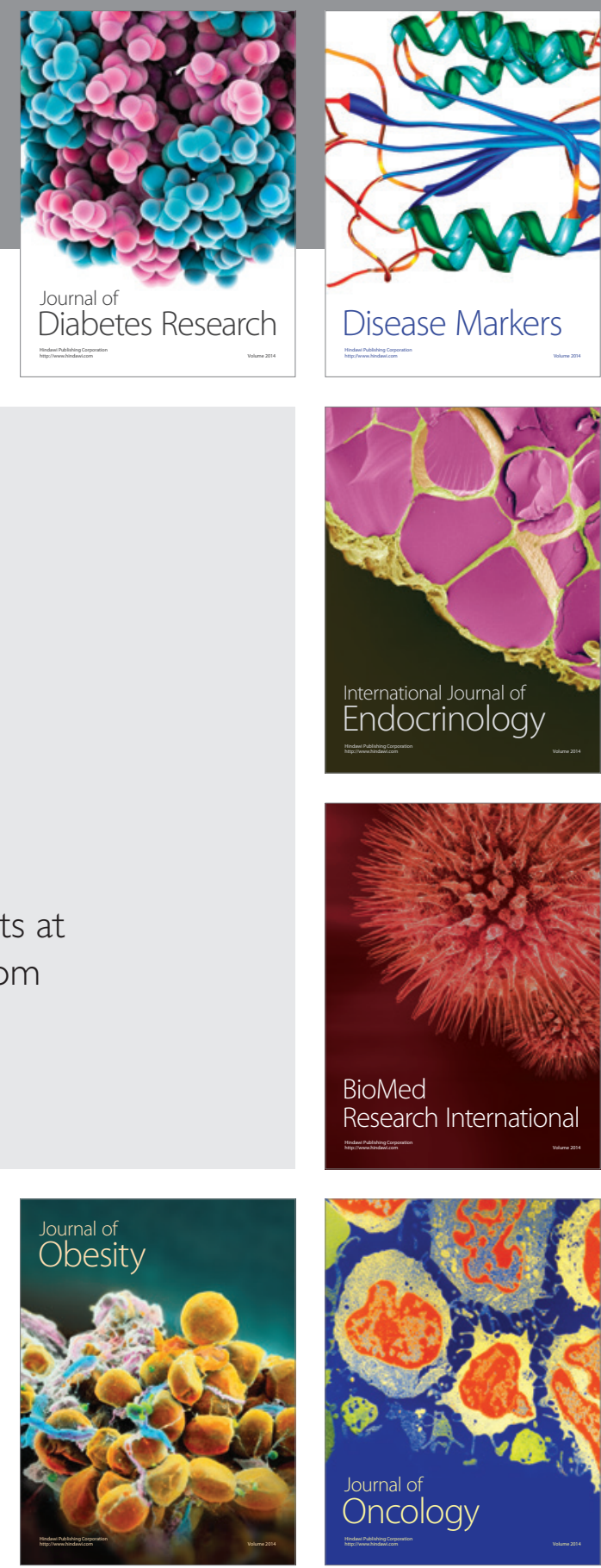

Disease Markers
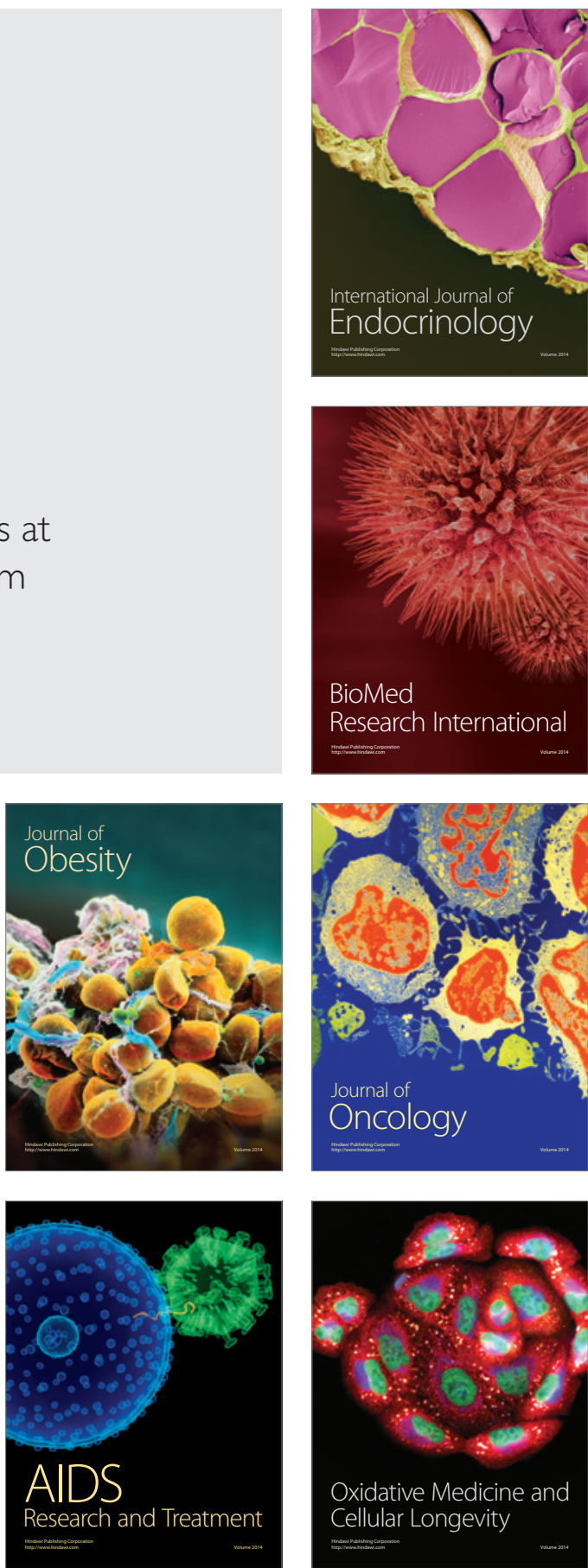